\title{
Hawking radiation and W-infinity algebra
}

\section{Bonora*}

International School for Advanced Studies (SISSA/ISAS)

Via Beirut 2-4, 34014 Trieste, Italy, and INFN, Sezione di Trieste

E-mail: bonoralsissa.it

\section{Cvitan}

Theoretical Physics Department, Faculty of Science, University of Zagreb Bijenička cesta 32, p.p. 331, HR-10002 Zagreb, Croatia

E-mail: mcvitan@phy.hr

\section{S. Pallua}

Theoretical Physics Department, Faculty of Science, University of Zagreb Bijenička cesta 32, p.p. 331, HR-10002 Zagreb, Croatia

E-mail: pallua@phy.hr

\section{Smolic}

Theoretical Physics Department, Faculty of Science, University of Zagreb Bijenička cesta 32, p.p. 331, HR-10002 Zagreb, Croatia

E-mail: ismolicephy.hr

\begin{abstract}
We review the calculation of the Hawking radiation in a Kerr black hole via the trace anomaly or the diff anomaly method. These methods account for the integrated radiation. But in order to describe the full Planckian spectrum of the Hawking radiation one needs to introduce higher spin currents. We show that the latter describe the higher moments of the Hawking radiation. This result is related to the appearance of higher order Schwarzian derivatives in the conformal transformation laws of these currents. We show that one can construct covariant higher spin currents and that such currents are covariantly conserved. In other words there are no anomalies in currents with spin higher than two. The higher spin currents form a $W_{1+\infty}$ algebra. We show that this algebraic structure is at the root of their predictivity and in accordance with the no hairs theorem. This hints at the existence of a symmetry larger than the conformal one in the near horizon region of a black hole.
\end{abstract}

5th International School on Field Theory and Gravitation, April 20 - 242009

Cuiabá city, Brazil

\footnotetext{
${ }^{*}$ Speaker.
} 


\section{Introduction}

This is a review of the results obtained in $[1,2,3]$ concerning the calculation of the Hawking radiation of a black hole with anomaly-related methods. These methods use in an essential way the effective reduction to two dimensions of the near-horizon dynamics of bosonic or fermionic matter fields in a black hole background, and the ensuing effective conformal invariance of the action. Under these circumstances one can use either the trace or the diffeomorphism anomaly method in order to compute the integrated Hawking radiation. The Hawking radiation $[4,5]$ does not depend on the details of the collapse that gives rise to a black hole. Therefore one expects that the methods to calculate it should have the same character of universality. The anomaly method has such characteristics. The first attempt to compute the Hawking radiation by exploiting trace anomalies was made by Christensen and Fulling, [6] (see also [7]), and re-proposed subsequently by $[8,9]$ in a modified form. More recently a renewed interest in the same problem has been raised by [10], which makes use of the diffeomorphisms anomaly. This last paper has been at the origin of a considerable activity, see [3] for references. As we shall see the trace and diff anomaly methods are strictly related. As one can easily realize, they both stem from the Virasoro algebra symmetry underlying the theory.

However this is not the end of the story. As already pointed out, the trace and diff anomaly methods apply to the calculation of the integrated Hawking radiation and do not describe its spectrum. But one of the most interesting features of the Hawking radiation is precisely its Planckian spectrum. The latter can be 'Fourier analyzed' and expressed in terms of its higher moments or fluxes. It is therefore of upmost interest to be able to describe not only the integrated Hawking radiation but also such fluxes. An interesting proposal was made by the authors of $[14,15,16,17]$, who attributed these fluxes to phenomenological higher spin currents, i.e. higher spin generalizations of the energy-momentum tensor.

In $[1,2,3]$ it was shown that such higher currents do describe the higher spin fluxes of the Hawking radiation. By analogy with the case of the overall Hawking radiation, one would be tempted to attribute such predictive power to trace or diff anomalies in the higher spin currents, but the main result of $[1,2,3]$ is that this is actually not due to anomalies, but rather the the underlying $W_{(1+) \infty}$ algebra structure of the near horizon effective theory. In fact it was shown in the same references that these higher spin currents cannot have neither trace nor diff anomalies (or, rather, that, if there are anomalies, they are trivial). In [1] and [2] the analysis was limited to bosonic higher spin currents, in [3] the analysis and the conclusions were extended to fermionic currents. The only difference between the two is that the bosonic theory is characterized by a $W_{\infty}$, while the fermionic theory by a $W_{1+\infty}$ algebra (the 1 stands for the extension of the $W_{\infty}$ algebra to include a U(1) current).

Therefore the generalization to higher fluxes is not conveyed by higher spin current anomalies but by some other features of the higher spin currents. It is well-known that both diff and trace anomaly in $2 \mathrm{~d}$ stem from the so-called Gelfand-Fuks (or Virasoro) cocycle, which is is constructed by means of a Schwarzian derivative. There are infinite many higher spin generalizations of the Schwarzian derivative. It is these higher Schwarzian derivatives, incorporated in the conformal transformation properties of the higher spin currents, that are at the origin of the higher Hawking radiation fluxes. On the other hand such higher Schwarzian derivatives, contrary to original lowest 
Schwarzian derivative, are totally disconnected from anomalies.

Now, the requirement to describe higher fluxes of the Hawking radiation does not uniquely determine by itself the higher spin currents. But if we add the request that the currents be anomaly free (as they must) we come to the conclusion that they must form a $W_{(1+) \infty}$ algebra. The main conclusion of our series of papers is that the Hawking radiation and, in particular its Planckian spectrum, points toward the existence in the near horizon region of a symmetry much larger than the Virasoro algebra, that is a $W_{\infty}$ or a $W_{1+\infty}$ algebra.

In this review we will start (section 2) from a Kerr-like metric in 4D and consider bosonic or fermionic matter coupled to the relevant background. We will review how to reduce the problem to two dimensions. Then the boson or spinor field will be expanded in the appropriate spherical harmonics. After integrating the action over the polar angles one is left with infinite many free two-dimensional boson or spinor fields interacting with the background gravity specified by the metric

$$
d s^{2}=f(r) d t^{2}-\frac{1}{f(r)} d r^{2}
$$

as well as to the electromagnetic field. $f(r)$ near the horizon behaves like $f(r) \approx 2 \kappa\left(r-r_{H}\right)$, where $\kappa$ is the surface gravity.

\section{Reduction to two dimensions}

In [3] we have shown that if the 4 dimensional action

$$
S=\int d^{4} x \sqrt{-g} \bar{\psi} \not \nabla
$$

represents a spinor in the background of a Kerr metric

$$
d s^{2}=\frac{\Delta}{\Sigma}\left(d t-a \sin ^{2} \theta d \phi\right)^{2}-\frac{\sin ^{2} \theta}{\Sigma}\left(a d t-\left(r^{2}+a^{2}\right) d \phi\right)^{2}-\left(r^{2}+a^{2} \cos ^{2} \theta\right)\left(\frac{d r^{2}}{\Delta}+d \theta^{2}\right)
$$

with $\left.\Sigma=r^{2}+a^{2} \cos ^{2} \theta, \Delta=r^{2}-2 M r+a^{2}\right)$ the near horizon $\left(r \approx r_{+}\right.$, where $r_{+}+r_{-}=2 M$ and $r_{+} r_{-}=a^{2}$ ) dynamics becomes effectively two-dimensional. This can be seen by expanding $\phi$ as $\psi=\sum_{l m} \psi_{l m}(t, r) S_{l m}(\theta) e^{-i m \phi}$, where the functions $S_{l m}$ are normalized so that $\int d \theta \sqrt{\Sigma} \sin \theta S_{l m}^{*}(\theta) S_{l^{\prime} m}(\theta)=$ $2 \delta_{l l^{\prime}}$. Integrating the action over $\theta$ and $\phi$ on finally gets

$$
S=\sum_{s=1}^{2} \sum_{l m} 4 \pi \int d t d r \Phi \bar{\chi}_{(s) l m} \not D \chi_{(s) l m}
$$

where the covariant derivative includes the gauge part $D_{\alpha}={ }^{(2)} \nabla_{\alpha}-i q A_{\alpha}$, and the charge $q$ of $\chi_{(s) l m}$ is $m$. This is the $2 D$ action for an infinite number of two components fermions $\chi_{(s) l m}$ in the background given by the dilaton $\Phi$

$$
\Phi=\sqrt{r^{2}+a^{2}},
$$

the gauge field $A_{\alpha}$

$$
A_{t}=\frac{e_{0}^{\phi}}{e_{0}{ }^{t}}=\frac{a}{r^{2}+a^{2}}, \quad A_{r}=0
$$


and the metric of the type (1.1)

$$
d s^{2}=f(r) d t^{2}-\frac{1}{f(r)} d r^{2}
$$

where

$$
f(r)=\frac{\Delta(r)}{r^{2}+a^{2}}, \quad \Delta=\left(r-r_{+}\right)\left(r-r_{-}\right)
$$

We are interested in the near horizon region. In this region the dilaton is approximately constant, so we may disregard it: the equations of motion are those of free fermions in two dimensions, coupled to the metric and the gauge field. This theory is conformal invariant.

A similar conclusion was obtained in [13] by considering a scalar field in 4D instead of a spinor: in the near horizon region the dynamics is described by infinite many two-dimensional scalar free fields in a background similar to the above one.

\section{The Diff and Trace anomaly method}

Having reduced the problem to two dimensions let us review now the two anomaly methods used to compute the integrated Hawking radiation. To start with we consider the simplified situation in which the electromagnetic background field is decoupled $(m=0)$. The method employed in [10] is based on the diffeomorphism anomaly in a two-dimensional effective field theory near the horizon of a radially symmetric static black hole. The basic argument is that, since just outside the horizon the ingoing modes cannot classically influence the physics outside the black hole, they can be integrated out, giving rise to an effective theory of purely outgoing modes. So the physics in that region can be described by an effective two-dimensional chiral field theory (of infinite many fields). This implies an effective breakdown of the diffeomorphism invariance. The ensuing anomaly equation can be utilized to compute the outgoing flux of radiation. The latter appears as the quantum agent that restores the diffeomorphism symmetry.

\subsection{Diff anomaly method}

Let us describe in detail the corresponding derivation as given, in a somewhat simplified form, in [11]. The range of $r$ contains two relevant regions: the region $o$, defined by $r>r_{H}+\epsilon, r_{H}$ being the horizon radius, and the region $H$, defined by $r_{H}<r<r_{H}+\epsilon$. The region $H$ is where the ingoing modes have been integrated out, therefore the effective field theory there is anomalous, while in $o$ we expect a fully symmetric theory. This is expressed by a vanishing energy momentum tensor covariant divergence

$$
\nabla_{\mu} T_{\nu(O)}^{\mu}=0
$$

while in the $H$ region we have

$$
\nabla_{\mu} T^{\mu}{ }_{\nu(H)}=\frac{\hbar c_{R}}{96 \pi} \epsilon_{\nu \mu} \partial^{\mu} R
$$

This is the covariant form of the diffeomorphism anomaly, with a coefficient appropriate for chiral (outgoing or right) matter with central charge $c_{R}$. In (3.2) $\epsilon_{\mu \nu}=\sqrt{-g} \varepsilon_{\mu \nu}$, where $\varepsilon$ is the numerical 
antisymmetric symbol $\left(\varepsilon_{01}=1\right)$. In the case of the background metric we are considering, the determinant is -1 . Since the metric is also static, the two equations above take, for $T_{t}^{r}$, a very simple form:

$$
\partial_{r} T_{t(o)}^{r}=0
$$

and

$$
\partial_{r} T_{t(H)}^{r}=\partial_{r} N_{t}^{r} \equiv \partial_{r}\left(\frac{\hbar c_{R}}{96 \pi}\left(f f^{\prime \prime}-\frac{1}{2}\left(f^{\prime}\right)^{2}\right)\right)
$$

respectively. Now we integrate these equations in the respective regions of validity

$$
T_{t(o)}^{r}=a_{o}
$$

and

$$
T_{t(H)}^{r}(r)=a_{H}+N_{t}^{r}(r)-N_{t}^{r}\left(r_{H}\right)
$$

We remark that $a_{o}$, being constant, determines (together with the condition that there is no ingoing flux from infinity) the outgoing energy flux. This is the quantity we would like to know. To this end we define the overall energy-momentum tensor.

$$
T_{t}^{r}=T_{t(o)}^{r} \theta\left(r-r_{H}-\epsilon\right)+T_{t(H)}^{r}\left(1-\theta\left(r-r_{H}-\epsilon\right)\right)
$$

It is understood that $\epsilon$ is a small number which specifies the size of the region where the energymomentum tensor is not conserved. If we take the divergence of (3.7), we get

$$
\partial_{r} T_{t}^{r}=\left(a_{o}-a_{H}+N_{t}^{r}\left(r_{H}\right)\right) \delta\left(r-r_{h}-\epsilon\right)+\partial_{r}\left(N_{t}^{r}(r) H(r)\right)
$$

where $H(r)=1-\theta\left(r-r_{H}-\epsilon\right)$. We can now define a new overall tensor

$$
\hat{T}_{t}^{r}(r)=T_{t}^{r}(r)-N_{t}^{r}(r) H(r)
$$

which is conserved

$$
\partial_{r} \hat{T}_{t}^{r}=0
$$

provided that

$$
a_{o}-a_{H}+N_{t}^{r}\left(r_{H}\right)=0
$$

Now, the condition that at the horizon the energy-momentum tensor vanishes, leads to $a_{H}=0$ (see (3.6)). Therefore

$$
a_{o}=N_{t}^{r}\left(r_{H}\right)=\frac{\hbar \kappa^{2}}{48 \pi} c_{R}
$$

This is the outgoing flux at infinity and coincides with the total Hawking radiation (see below) emitted by the black hole specified by the metric (1.1). We remark that $\hat{T}_{t}^{r}$ is constant everywhere. 


\subsection{Trace anomaly method}

The method based on the trace anomaly was put forward long ago by Christensen and Fulling, [6] (see also [7]) and has been re-proposed in different forms in [8, 9] and, in particular, in [15] and [17] (see also [1,2]). This approach is based on the argument that the near-horizon physics is described by a two-dimensional conformal field theory (see above). Classically the trace of the matter energy momentum tensor vanishes on shell. However it is generally nonvanishing at one loop, due to the anomaly: $T_{\alpha}^{\alpha}=\frac{c}{48 \pi} R$, where $R$ is the background Ricci scalar. $c$ is the total central charge of the matter system. The idea is to use this piece of information in order to compute the same constant $a_{o}$ calculated with the previous method. Here we do not have to split the space in different regions, but we consider a unique region outside the horizon.

With reference to the metric (1.1) it is convenient to transform it into a conformal metric. This is done by means of the 'tortoise' coordinate $r_{*}$ defined via $\frac{\partial r}{\partial r_{*}}=f(r)$. Next it is useful to introduce light-cone coordinates $u=t-r_{*}, v=t+r_{*}$. Let us denote by $T_{u u}(u, v)$ and $T_{v v}(u, v)$ the classically non vanishing components of the energy-momentum tensor in these new coordinates. Our black hole is now characterized by the background metric $g_{\alpha \beta}=e^{\varphi} \eta_{\alpha \beta}$, where $\varphi=\log f$. The energy-momentum tensor can be calculated by integrating the conservation equation and using the trace anomaly. The result is (see below)

$$
T_{u u}(u, v)=\frac{\hbar c_{R}}{24 \pi}\left(\partial_{u}^{2} \varphi-\frac{1}{2}\left(\partial_{u} \varphi\right)^{2}\right)+T_{u u}^{(h o l)}(u)
$$

where $T_{u u}^{(h o l)}$ is holomorphic, while $T_{u u}$ is conformally covariant. Namely, under a conformal transformation $u \rightarrow \tilde{u}=f(u)(v \rightarrow \tilde{v}=g(v))$ one has

$$
T_{u u}(u, v)=\left(\frac{d f}{d u}\right)^{2} T_{\tilde{u} \tilde{u}}(\tilde{u}, v)
$$

Since, under a conformal transformation, $\tilde{\varphi}(\tilde{u}, \tilde{v})=\varphi(u, v)-\ln \left(\frac{d f}{d u} \frac{d g}{d v}\right)$, it follows that

$$
T_{\tilde{u} \tilde{u}}^{(h o l)}(\tilde{u})=\left(\frac{d f}{d u}\right)^{-2}\left(T_{u u}^{(h o l)}(u)+\frac{\hbar c_{R}}{24 \pi}\{\tilde{u}, u\}\right)
$$

where $\{\tilde{u}, u\}$ denotes the Schwarzian derivative. Near the horizon good coordinates are the Kruskal ones, $(U, V)$, defined by $U=-e^{-\kappa u}$ and $V=e^{\kappa v}$. Under this transformation we have

$$
T_{U U}^{(h o l)}(U)=\left(\frac{1}{\kappa U}\right)^{2}\left(T_{u u}^{(h o l)}(u)+\frac{\hbar c_{R}}{24 \pi}\{U, u\}\right)
$$

Now we require the outgoing energy flux to be regular at the future horizon $U=0$ in the Kruskal coordinate. Therefore at that point $T_{u u}^{(h o l)}(u)$ is given by $\frac{c_{R} \kappa^{2}}{48 \pi}$. We remark that this implies in particular that $T_{u u}\left(r=r_{H}\right)=0$.

Since the background is static, $T_{u u}^{(h o l)}(u)$ is constant in $t$ and therefore also in $r$. So at $r=\infty$ it takes the same value $\frac{\hbar c_{R} \kappa^{2}}{48 \pi}$. On the other hand we can assume that at $r=\infty$ there is no incoming flux and that the background is trivial (so that the vev of $T_{u u}^{(h o l)}(u)$ and $T_{u u}(u, v)$ asymptotically coincide). 
Finally the asymptotic flux is

$$
\left\langle T_{t}^{r}\right\rangle=\left\langle T_{u u}\right\rangle-\left\langle T_{v v}\right\rangle=\frac{\hbar \kappa^{2}}{48 \pi} c_{R}
$$

This outgoing flux coincides with the constant $a_{o}$ calculated above.

Summarizing we can say that the basic ingredients of the two methods are:

- (a) in the first case the integration of the anomalous and non-anomalous conservation of the energy-momentum tensor, in the second case the integration of the energy-momentum conservation in the presence of a trace anomaly;

- (b) in both cases we have the condition that the energy-momentum tensor vanishes at the horizon and there is no incoming energy flux from infinity.

What energy-momentum tensor vanishes at the horizon will be clarified below.

\subsection{Comparison between the two methods}

The generic case of a chiral two-dimensional theory with central charge $c_{R}$ and $c_{L}$ for the holomorphic and anti-holomorphic part, respectively, is characterized by the presence of both diffeomorphism and trace anomaly, i.e.

$$
\nabla_{\mu} T_{\nu}^{\mu}=\frac{\hbar}{48 \pi} \frac{c_{R}-c_{L}}{2} \epsilon_{\nu \mu} \partial^{\mu} R
$$

and

$$
T_{\alpha}^{\alpha}=\frac{\hbar}{48 \pi}\left(c_{R}+c_{L}\right) R
$$

Let us rewrite these equations in terms of the light-cone coordinates $u$ and $v$ introduced before. In this basis the nonvanishing metric elements take the form:

$$
g_{u v}=\frac{1}{2} e^{\varphi}=-\epsilon_{u v}, \quad g^{u v}=2 e^{-\varphi}=\epsilon^{u v}
$$

and eq.(3.18) becomes

$$
\begin{aligned}
& \nabla_{u} T_{u v}+\nabla_{v} T_{u u}=\frac{\hbar}{48 \pi} \frac{c_{R}-c_{L}}{2} \epsilon_{u v} \partial_{u} R \\
& \nabla_{u} T_{v v}+\nabla_{v} T_{u v}=-\frac{\hbar}{48 \pi} \frac{c_{R}-c_{L}}{2} \epsilon_{u v} \partial_{v} R
\end{aligned}
$$

On the other hand (3.19) becomes

$$
T_{u v}=\frac{\hbar}{48 \pi} \frac{c_{R}+c_{L}}{4} R e^{\varphi}
$$

Replacing this with $R=-4 \partial_{u} \partial_{v} \varphi e^{-\varphi}$ in (3.22), we get

$$
\partial_{v} T_{u u}=\frac{\hbar}{24 \pi} c_{R} \partial_{v} \mathcal{T}_{u u}
$$


where

$$
\mathcal{T}_{u u}=\partial_{u}^{2} \varphi-\frac{1}{2}\left(\partial_{u} \varphi\right)^{2}
$$

Integrating (3.24) we get

$$
T_{u u}(u, v)=\frac{\hbar}{24 \pi} c_{R} \mathcal{T}_{u u}(u, v)+T_{u u}^{(h o l)}(u)
$$

where $T_{u u}^{(h o l)}$ depends only on $u$.

Similarly, integrating (3.21), one obtains

$$
T_{v v}(u, v)=\frac{\hbar}{24 \pi} c_{L} \mathcal{T}_{v v}(u, v)+T_{v v}^{(a-h o l)}(v)
$$

where $\mathcal{T}_{v v}=\partial_{v}^{2} \varphi-\frac{1}{2}\left(\partial_{v} \varphi\right)^{2}$, and $T_{v v}^{(a-h o l)}$ depends only on $v$. The two equations (3.26) and (3.27) are our basic result. They are equivalent to the two equations (3.18) and (3.19).

In the "trace anomaly" method we have utilized eq.(3.26), required that the energy-momentum tensor be conserved and imposed the conditions (b) of the previous section. This, in particular, amounts to requiring $c_{R}=c_{L}$ in the region outside the horizon. We see now that the possibility to integrate (3.18) in the presence of (3.19) is actually insensitive to the relation between $c_{L}$ and $c_{R}{ }^{1}$.

In the "diff anomaly" approach we integrated (3.18) in the near horizon region and the conserved energy-momentum divergence away from the horizon. Then we imposed vanishing of energy-momentum tensor at the horizon. It is obvious that we used again (3.26) and (3.27) in disguise.

It is actually possible to be more specific. We have already noticed that in the trace anomaly method $T_{u u}\left(r=r_{H}\right)=0$. On the other hand we point out that $T_{v v}^{(a-h o l)}$ is constant in $r$ and $t$, for the same reason as $T_{u u}^{(h o l)}$ is, and thus vanishes upon the request of no ingoing flux from infinity. It is also easy to see that, if $c_{R}=c_{L}, \mathcal{T}_{u u}=\mathcal{T}_{v v}$. Therefore $T_{t}^{r}=T_{u u}-T_{v v}$ is constant everywhere and equals the outgoing flux (3.17) at infinity. Therefore the $T_{t}^{r}$ of subsection 2.2 equals $\hat{T}_{t}^{r}$ of subsection 2.1. And it is also clear that the energy-momentum tensor vanishing at the horizon in subsection 2.1 is to be compared with $T_{u u}(u, v)$ of subsection 2.2.

It was important to stress the basic role of (3.26) and (3.27) because, as we will see, when we come to higher spin currents, it is not possible to describe the higher flux moments by means of anomalies (either trace or diff), but the analogues of (3.26) and (3.27) still hold and provide the desired description.

\section{Higher moments of the Hawking radiation}

The bosonic spectrum of the black hole is given by the Planck distribution

$$
N(\omega)=\frac{g_{*}}{e^{\beta \omega}-1}
$$

\footnotetext{
${ }^{1}$ In other words we can integrate the trace anomaly even if $c_{R} \neq c_{L}$. This is clearly only a characteristic of two dimensions
} 
where $1 / \beta$ is the Hawking temperature and $\omega=|k|$, the absolute value of the momentum. $g_{*}$ is the number of physical degrees of freedom in the emitted radiation. In two dimensions we can define the flux moments as follows

$$
F_{n}^{B}=\frac{g_{*}}{4 \pi} \int_{-\infty}^{+\infty} d k \frac{\omega k^{n-2}}{e^{\beta \omega}-1}
$$

They vanish for $n$ odd, while for $n$ even they are given by

$$
F_{2 n}^{B}=\frac{1}{2 \pi} \int_{0}^{\infty} d \omega \omega^{2 n-1} N(\omega)=g_{*} \frac{(-1)^{n+1}}{8 \pi n} B_{2 n} \kappa^{2 n}
$$

where $B_{n}$ are the Bernoulli numbers $\left(B_{2}=\frac{1}{6}, B_{4}=-\frac{1}{30}, ..\right)$. Therefore the outgoing flux (3.17) is seen to correspond to $F_{2}^{B}$ when $g_{*}=c_{R}$. $F_{2}^{B}$ is the total integrated outgoing flux.

The fermionic spectrum of the Kerr black hole (per degree of freedom) is given by the Planck distribution

$$
N(\omega)=\frac{1}{e^{\beta(\omega-m \Omega)}+1}
$$

where $\Omega$ is the total angular momentum, in our case $\Omega=A_{t}$ evaluated at the horizon and $m$ is the charge.

Let us consider first the case $m=0$. In two dimensions we can define the flux moments $F_{n}$, which vanish for $n$ odd, while for $n$ even they are given by, [18],

$$
F_{2 n}=\frac{1}{2 \pi} \int_{0}^{\infty} d \omega \frac{\omega^{2 n-1}}{e^{\beta \omega}+1}=\frac{\kappa^{2 n} B_{2 n}}{4 \pi n}\left(1-2^{1-2 n}\right)(-1)^{n+1}
$$

where $B_{s}$ 's are the Bernoulli numbers $\left(B_{2}=1 / 6, B_{4}=-1 / 30, \ldots\right)$ and $\kappa=2 \pi / \beta$ is the surface gravity of the black hole.

When $m \neq 0$ we do not have similar compact formulas, however it makes sense to sum over the emission of a particle (with charge $m$ ) and the corresponding antiparticle (with charge $-m$ ). In this case the flux moments become

$$
\begin{aligned}
F_{n+1}^{\Omega} & =\frac{1}{2 \pi}\left(\int_{0}^{\infty} d x \frac{x^{n}}{e^{\beta(x-m \Omega)}+1}-(-1)^{n} \int_{0}^{\infty} d x \frac{x^{n}}{e^{\beta(x+m \Omega)}+1}\right) \\
& =\frac{(m \Omega)^{n+1}}{2 \pi(n+1)}-\sum_{k=1}^{[(n+1) / 2]}(-1)^{k} \frac{n !\left(1-2^{1-2 k}\right) \kappa^{2 k}}{2 \pi(2 k) !(n+1-2 k) !} B_{2 k}(m \Omega)^{n+1-2 k}
\end{aligned}
$$

Unlike the bosonic case, once we know $F_{n}^{\Omega}$ we do not have enough information to reconstruct the full spectrum with $m \neq 0$, but if one is able to reproduce the moments $F_{n}^{\Omega}$ it is anyhow an important positive test.

\section{A $W_{(1+) \infty}$ algebra and higher spin currents}

In order to derive the higher Hawking fluxes we postulate the existence of conserved higher spin spin currents. The latter can be either bosonic or fermionic. In $[1,2]$ the case of currents constructed out of a chiral bosonic field was dealt with, while in [3] we considered currents bilinear made of chiral fermions of the $2 \mathrm{D}$ effective field theory near the horizon. These currents will play 
a role analogous to the energy-momentum tensor for the integrated radiation (the lowest moment). For reasons of brevity here we limit ourselves to reviewing the case of fermionic currents.

To construct the fermionic currents we start from a $W_{1+\infty}$ algebra defined in an abstract flat space spanned by a local coordinate $z$. These currents were introduced in [12]:

$$
\begin{gathered}
j_{z \ldots z}^{(s)}(z)=-\frac{B(s)}{s} \sum_{k=1}^{s}(-1)^{k}\left(\begin{array}{l}
s-1 \\
s-k
\end{array}\right)^{2}: \partial_{z}^{s-k} \Psi^{\dagger}(z) \partial_{z}^{k-1} \Psi(z): \\
B(s) \equiv \frac{2^{s-3} s !}{(2 s-3) ! !} q^{s-2} \quad s=1,2,3, \ldots
\end{gathered}
$$

where $q$ is a deformation parameter. These currents form a $W_{1+\infty}$ algebra.

The spin $s$ currents $j_{z \ldots z}^{(s)}(z)$ are linear combinations of bilinears

$$
j_{z \ldots z}^{(m, n)}(z) \equiv: \partial^{m} \Psi^{\dagger} \partial^{n} \Psi: \equiv \lim _{z_{1}, z_{2} \rightarrow z}\left(\partial_{z_{1}}^{m} \Psi^{\dagger}\left(z_{1}\right) \partial_{z_{2}}^{n} \Psi\left(z_{2}\right)-\partial_{z_{1}}^{m} \partial_{z_{2}}^{n}\left\langle\Psi^{\dagger}\left(z_{1}\right) \Psi\left(z_{2}\right)\right\rangle\right)
$$

We wish to relate the currents written in two different coordinate systems, connected by coordinate change $z \rightarrow w(z)$. That is, our aim is to obtain a relation analogous to the one found for the energy momentum tensor,

$$
j_{z \ldots z}^{(s)}(z) \rightarrow\left(\frac{1}{\kappa w}\right)^{s}\left(j_{z \ldots z}^{(s)}+\left\langle X_{s}^{F}\right\rangle\right)
$$

and specify this to a transformation $w(z)=-e^{-\kappa z}$ so as to obtain the value of $j_{z \ldots z}^{(s)}(z)$ at the horizon by requiring regularity.

Using the transformation property of holomorphic fermionic field

$$
\Psi(z)=\left(w^{\prime}(z)\right)^{1 / 2} \Psi(w)
$$

and

$$
\begin{aligned}
& : \partial_{z_{1}}^{m} \Psi^{\dagger}\left(z_{1}\right) \partial_{z_{2}}^{n} \Psi\left(z_{2}\right):=\partial_{z_{1}}^{m} \partial_{z_{2}}^{n}\left(\left(w_{1}^{\prime}\left(z_{1}\right)\right)^{1 / 2}\left(w_{2}^{\prime}\left(z_{2}\right)\right)^{1 / 2}: \Psi^{\dagger}\left(w_{1}\right) \Psi\left(w_{2}\right):\right) \\
& +\partial_{z_{1}}^{m} \partial_{z_{2}}^{n}\left(\left(w_{1}^{\prime}\left(z_{1}\right)\right)^{1 / 2}\left(w_{2}^{\prime}\left(z_{2}\right)\right)^{1 / 2}\left\langle\Psi^{\dagger}\left(w_{1}\right) \Psi\left(w_{2}\right)\right\rangle-\left\langle\Psi^{\dagger}\left(z_{1}\right) \Psi\left(z_{2}\right)\right\rangle\right)
\end{aligned}
$$

where

$$
\left\langle\Psi^{\dagger}(z) \Psi(w)\right\rangle=\frac{\lambda}{z-w}
$$

is the propagator of the fermionic field and $\lambda$ a constant to be determined, one finds

$$
\left\langle X_{s}^{F}\right\rangle=-\lambda \frac{\kappa^{s} B_{s}}{s}\left(1-2^{1-s}\right)(4 q)^{s-2}=-\left\langle j_{z \ldots z}^{(s)}\right\rangle_{h}
$$

where $\langle\cdot\rangle_{h}$ denotes the value at the horizon. Notice that $\left\langle X_{s}^{F}\right\rangle=0$ for an odd spin $s$. For $s>1$ this is because $B_{s}=0$ for odd $s>1$. For $s=1$ it is because of the other factor in (5.5). The value of $\lambda$ is determined in such a way as to reproduce the transformation properties of the energy-momentum tensor and, in physical units, is proportional to $\hbar$. Eventually we will set $\lambda=\hbar$. 


\section{Higher spin covariant currents}

The holomorphic currents of the previous section are defined on a background with a trivial Euclidean metric. But we need currents defined in the appropriate non trivial background that characterizes a Kerr black hole. In order to construct the covariant higher-spin currents from fermionic fields in such background, we will follow [17]. First we recall some properties of fermions in two dimensions. The equation of motion for a right-handed fermion with unit charge is given by

$$
\left(\partial_{u}-i A_{v}+\frac{1}{4} \partial_{v} \varphi\right) \psi(u, v)=0
$$

In the Lorentz gauge, the gauge field can be written locally as $A_{u}=\partial_{u} \eta(u, v)$ and $A_{v}=-\partial_{v} \eta(u, v)$ where $\eta(u, v)$ is a scalar field. Since gravitational and gauge fields are not generally holomorphic, $\psi(u, v)$ is not holomorphic either. In order to construct holomorphic quantities from a fermionic field, we define a new field $\Psi$ by

$$
\Psi \equiv \exp \left(\frac{1}{4} \varphi(u, v)+i \eta(u, v)\right) \psi(u, v)
$$

It is easy to show that the equation of motion implies $\partial_{v} \Psi=0$ and hence $\Psi$ is holomorphic. Similarly we can define $\Psi^{\dagger}$ as

$$
\Psi^{\dagger} \equiv \exp \left(\frac{1}{4} \varphi(u, v)-i \eta(u, v)\right) \psi^{\dagger}(u, v)
$$

The equation of motion again guarantees that $\partial_{v} \Psi^{\dagger}=0$, so that $\Psi^{\dagger}$ is also holomorphic. We will use $\Psi$ and $\Psi^{\dagger}$ as the basic chiral fields to construct the $W_{1+\infty}$ algebra introduced in the previous section. To covariantize the expressions of the currents we reduce the problem to one dimension by considering only the $u$ dependence and keeping $v$ fixed. In one dimension a curved coordinate $u$ in the presence of a background metric

$$
g_{\mu \nu}=e^{\varphi(u, v)} \eta_{\mu \nu}
$$

is easily related to the corresponding normal coordinate $x$ by the equation $\partial_{x}=e^{-\varphi(u, v)} \partial_{u}$. We view $u$ as $u(x)$ and, by the above equation, we extract the correspondence between $j_{z \ldots z}^{(s)}$ and $j_{u \ldots u}^{(s)}$ by identifying $u$ with the coordinate $z$ of the previous section after Wick rotation. The expressions we get in this way are not yet components of the covariant currents. We have to remember the current conformal weights and introduce suitable factors in order to take them into account.

Under a holomorphic conformal transformation $u \rightarrow \tilde{u}$ the function $\varphi(u, v)$ and the field $\Psi(u)$ transform according to

$$
\begin{aligned}
\tilde{\varphi}(\tilde{u}, v) & =\varphi(u, v)-\ln \left(\frac{d \tilde{u}}{d u}\right) \\
\tilde{\Psi}(\tilde{u}) & =\left(\frac{d \tilde{u}}{d u}\right)^{\frac{1}{2}} \Psi(u)
\end{aligned}
$$

Therefore $e^{-\varphi / 2} \Psi(u)$ (and analogously, $e^{-\varphi / 2} \Psi^{\dagger}(u)$ ) transforms as a scalar with respect to a holomorphic coordinate transformation. 
As a consequence the covariant derivative of $\Psi(u)$ turns out to be

$$
\begin{gathered}
\nabla_{u} \Psi(u)=\left(\partial_{u}-\frac{1}{2} \partial_{u} \varphi-2 i A_{u}\right) \Psi(u) \\
\nabla_{u} \Psi^{\dagger}(u)=\left(\partial_{u}-\frac{1}{2} \partial_{u} \varphi+2 i A_{u}\right) \Psi^{\dagger}(u)
\end{gathered}
$$

and for higher covariant derivatives we have,

$$
\begin{aligned}
\nabla_{u}^{m+1} \Psi(u) & =\left(\partial_{u}-\left(m+\frac{1}{2}\right) \partial_{u} \varphi-2 i A_{u}\right) \nabla_{u}^{m} \Psi(u) \\
\nabla_{u}^{m+1} \Psi^{\dagger}(u) & =\left(\partial_{u}-\left(m+\frac{1}{2}\right) \partial_{u} \varphi+2 i A_{u}\right) \nabla_{u}^{m} \Psi^{\dagger}(u)
\end{aligned}
$$

It can be shown that $e^{-\left(m+\frac{1}{2}\right) \varphi} \nabla_{u}^{m} \Psi(u)$ and $e^{-\left(m+\frac{1}{2}\right) \varphi} \nabla_{u}^{m} \Psi^{\dagger}(u)$ transform as scalars under holomorphic coordinate transformation, for every $m \in \mathbb{N}$.

After these preliminaries the covariant currents are constructed using the following bricks:

$$
\begin{aligned}
J_{u \ldots u}^{(m, n)}= & e^{(m+n+1) \varphi(u, v)} \lim _{\epsilon \rightarrow 0}\left(e^{2 i \int_{u_{-}}^{u_{+}} A_{u}\left(u^{\prime}, v\right) d u^{\prime}}\right. \\
& \left.e^{-(m+1 / 2) \varphi\left(u_{+}, v\right)} \nabla_{u}^{m} \Psi^{\dagger}\left(u_{+}\right) e^{-(n+1 / 2) \varphi\left(u_{+}, v\right)} \nabla_{u}^{n} \Psi\left(u_{-}\right)-\frac{c_{m, n}^{f}}{\epsilon^{m+n+1}}\right)
\end{aligned}
$$

where we have used the abbreviations $u_{+} \equiv u(x+\epsilon / 2)$ and $u_{-} \equiv u(x-\epsilon / 2)$. The numerical constants $c_{m, n}^{f}$, defined by

$$
c_{m, n}^{f}=\lambda(-1)^{m}(m+n) !
$$

are determined in such a way that all singularities are canceled in the final expressions for $J^{(m, n)}$.

Finally, let us define the covariant currents corresponding to the $W_{1+\infty}$ fermionic currents:

$$
\begin{aligned}
& J_{u \ldots u}^{(s)}=-\frac{B(s)}{s} \sum_{k=1}^{s}(-1)^{k}\left(\begin{array}{l}
s-1 \\
s-k
\end{array}\right)^{2} J_{u \ldots u}^{(s-k, k-1)} \\
& B(s) \equiv \frac{2^{s-3} s !}{(2 s-3) ! !} q^{s-2}
\end{aligned}
$$

The first few covariant $W_{1+\infty}$ fermionic currents can be written in pretty simple form, using 
the abbreviation $T \equiv \partial_{u}^{2} \varphi-\frac{1}{2}\left(\partial_{u} \varphi\right)^{2}$

$$
\begin{aligned}
J_{u}^{(1)}= & j_{u}^{(1)}+\frac{i \lambda}{2 q} A_{u} \\
J_{u u}^{(2)}= & \left(2 A_{u}^{2}-\frac{T}{12}\right) \lambda-2 A_{u} J_{u}^{(1)}+j_{u u}^{(2)} \\
J_{u u u}^{(3)}= & -4 J_{u}^{(1)} A_{u}^{2}-4 J_{u u}^{(2)} A_{u}+\left(\frac{8 A_{u}^{3}}{3}-\frac{A_{u} T}{3}\right) \lambda+\frac{T J_{u}^{(1)}}{6}+j_{u u u}^{(3)} \\
J_{\text {uиuи }}^{(4)}= & +\lambda\left(4 A_{u}^{4}-\frac{7 T A_{u}^{2}}{5}-\frac{2}{5}\left(\nabla_{u}^{2} A_{u}\right) A_{u}+\frac{7 T^{2}}{240}+\frac{3}{5}\left(\nabla_{u} A_{u}\right)^{2}\right) \\
& -8 J_{u}^{(1)} A_{u}^{3}-12 J_{u u}^{(2)} A_{u}^{2}+\left(\frac{1}{5} \nabla_{u}^{2} J_{u}^{(1)}+\frac{7 T J_{u}^{(1)}}{5}-6 J_{u u u}^{(3)}\right) A_{u}-\frac{3}{5}\left(\nabla_{u} A_{u}\right)\left(\nabla_{u} J_{u}^{(1)}\right) \\
& +\frac{1}{5}\left(\nabla_{u}^{2} A_{u}\right) J_{u}^{(1)}+\frac{7 T J_{u u}^{(2)}}{10}+j_{u u u u}^{(4)}
\end{aligned}
$$

For higher order currents see [2].

Using these explicit expressions of the currents $J^{(s)}$,one can write down their covariant derivatives,

$$
\begin{aligned}
g^{u v} \nabla_{v} J_{u}^{(1)}= & -\lambda F_{u}{ }^{u} \\
g^{u v} \nabla_{v} J_{u u}^{(2)}= & \frac{1}{24} \lambda\left(\nabla_{u} R\right)+F_{u}{ }^{u} J_{u}^{(1)} \\
g^{u v} \nabla_{v} J_{u u u}^{(3)}= & 2 F_{u}{ }^{u} J_{u u}^{(2)}-\frac{1}{12}\left(\nabla_{u} R\right) J_{u}^{(1)} \\
g^{u v} \nabla_{v} J_{u u u u}^{(4)}= & \frac{3}{10}\left(\nabla_{u} F_{u}{ }^{u}\right)\left(\nabla_{u} J_{u}^{(1)}\right)-\frac{1}{10} F_{u}{ }^{u}\left(\nabla_{u}^{2} J_{u}^{(1)}\right)-\frac{1}{10}\left(\nabla_{u}^{2} F_{u}{ }^{u}\right) J_{u}^{(1)} \\
& -\frac{7}{20}\left(\nabla_{u} R\right) J_{u u}^{(2)}+3 F_{u}{ }^{u} J_{u u u}^{(3)}
\end{aligned}
$$

In the case of lowest spin current, $J^{(1)},(6.11)$ gives rise to the gauge anomaly

$$
g^{\mu \nu} \nabla_{\mu} J_{\nu}^{(1)}=-\frac{\hbar}{2} \epsilon^{\mu \nu} F_{\mu \nu}
$$

Apart from the gauge anomaly in the first current we are interested to check whether there are trace anomalies in the other currents. This is done as follows. After the RHS of the above equation is expressed in terms of covariant quantities, terms proportional to $\hbar$ (which is present only in $\lambda$ ) are identified as possible anomalies by proceeding in analogy to the energy-momentum tensor. One assumes that there is no anomaly in the conservation laws of covariant currents, that is that the covariant derivatives of the higher spin currents with the addition of suitable covariant terms (these terms are classical i.e. not proportional to $\hbar$, see for instance the terms in the LHS of $(6.12,6.13)$ ) vanish. Since

$$
(\nabla \cdot J)_{u \ldots u}+\ldots=g^{u v} \nabla_{v} J_{u \ldots u}+g^{u v} \nabla_{u} J_{v u \ldots u}+\ldots=0,
$$

where dots denote the above mentioned classical covariant terms, one relates terms proportional to $\hbar$ in the $u$ derivative of the trace ( $v u \ldots u$ components) to the terms proportional to $\hbar$ in the $v$ derivative of ${ }_{u \ldots u}$ components of the currents. 
For the covariant energy momentum tensor, $J^{(2)}$ we have $\operatorname{Tr}\left(J^{(2)}\right)=2 g^{v u} J_{v u}^{(2)}=-\frac{\hbar}{12} R$ which is the well known trace anomaly. In the case of $J^{(3)}$ current the terms that carry explicit factors of $\hbar$ cancel out in $g^{u v} \nabla_{v} J_{u u u}^{(3)}$, which implies absence of $\hbar$ in the trace, and consequently the absence of the trace anomaly. The same is true for $J^{(4)}$ and the higher currents.

In conclusion anomalies make their appearance only in the $J^{(1)}$ and $J^{(2)}$ currents.

\subsection{Higher moments of the Hawking radiation}

After constructing the higher spin currents let us come to the description of the higher moments of the fermionic Hawking radiation. We will follow the pattern outlined in section 3 and consider first the case in which the electromagnetic field is decoupled $(m=0)$.

In section 5 we evaluated $\left\langle j_{z \ldots z}^{(s)}\right\rangle_{h}$. If we identify $j_{z \ldots z}^{(s)}(z)$ via a Wick rotation with $j_{u \ldots u}^{(s)}(u)$ we get the corresponding value at the horizon, $\left\langle j_{u \ldots u}^{(s)}\right\rangle_{h}$. We notice that since the problem we are considering is stationary and $j_{u \ldots u}^{(s)}(u)$ is chiral, it follows that it is constant in $t$ and $r$. Therefore $\left\langle j_{u \ldots u}^{(s)}\right\rangle_{h}$ corresponds to its value at $r=\infty$. Since $j_{u \ldots u}^{(s)}(u)$ and $J_{u \ldots u}^{(s)}(u)$ asymptotically coincide, the asymptotic flux of these currents is

$$
\left\langle J^{(s)^{r}}{ }_{t \ldots t}\right\rangle=\left\langle J_{u \ldots u}^{(s)}\right\rangle-\left\langle J_{v \ldots v}^{(s)}\right\rangle=\left\langle j_{u \ldots u}^{(s)}\right\rangle_{h}
$$

If we set $q=\frac{i}{4}$ and $\lambda=1$ in conventional units and, as in [1,2] we multiply the currents by $-\frac{1}{2 \pi}$ in order to properly normalize the (physical) energy-momentum tensor, we get

$$
-\frac{1}{2 \pi}\left\langle J^{(2 n)^{r}} t \ldots t\right\rangle=-(-1)^{n} \frac{\kappa^{2 n} B_{2 n}}{4 \pi n}\left(1-2^{1-2 n}\right)
$$

while the odd currents give a vanishing value. These values correspond precisely to the fluxes of the Hawking Planckian spectrum defined by (4.3) multiplied by two. This is so because our currents carry both particle and antiparticle contributions.

Next we wish to take into account the presence of the gauge field, which, in our case, vanishes at infinity but not at the horizon. This introduces a significant change in our method. In section 3 the basic criterion was to require regularity of $T_{u u}^{(h o l)}$ at the horizon. Now the presence of the electromagnetic field interferes with the regularity of $T_{u u}^{(h o l)}$ at the horizon. As a consequence this criterion must be updated.

Let us start with the first current (6.7). From now on we understand that the electromagnetic field $A_{u}$ absorbs also the charge $m$, so that in the final results the replacement $A_{t} \rightarrow m A_{t}$ is understood. We easily get (remember that $\left\langle X_{1}^{F}\right\rangle$ vanishes)

$$
J_{\tilde{u}}^{(1)}=j_{\tilde{u}}^{(1)}+\frac{i \lambda}{2 q} A_{\tilde{u}}=\frac{1}{f_{u}}\left(j_{u}^{(1)}+\frac{i \lambda}{2 q} A_{u}\right)
$$

where $f_{u}$ denotes the first derivative of $f(u)=\tilde{u}$ with respect to $u$. Now let us introduce the Kruskal coordinate $f(u) \equiv U=-e^{-\kappa u}$. It is evident that we have to require regularity at the horizon of $j_{\tilde{u}}^{(1)}+\frac{i \lambda}{2 q} A_{\tilde{u}}$, not of $j_{\tilde{u}}^{(1)}$ alone. Therefore we get

$$
\left\langle j_{\tilde{u}}^{(1)}\right\rangle_{h}+\frac{i \lambda}{2 q}\left\langle A_{\tilde{u}}\right\rangle_{h}=0
$$


where $\langle\cdot\rangle_{h}$ denotes the value at the horizon. Now $j_{u}^{(1)}(u)$ is constant in $t$ and $r$. Therefore $\left\langle j_{u}^{(1)}\right\rangle_{h}=$ $-\frac{i \lambda}{2 q}\left\langle A_{u}\right\rangle_{h}$ corresponds to its value at $r=\infty$. Since $j_{u}^{(1)}(u)$ and $J_{u}^{(1)}(u)$ asymptotically coincide, because $A_{u}(u)$ asymptotically vanishes, we get

$$
\begin{aligned}
-\frac{1}{2 \pi}\left\langle J^{(1) r}\right\rangle & =-\frac{1}{2 \pi}\left\langle J_{u}^{(1)}\right\rangle+\frac{1}{2 \pi}\left\langle J_{v}^{(1)}\right\rangle \\
& =\frac{i \lambda}{4 \pi q}\left\langle A_{u}\right\rangle_{h}=\frac{1}{2 \pi} A_{t}=\frac{\Omega}{2 \pi}
\end{aligned}
$$

where $\langle\cdot\rangle$ represents the asymptotic value and we have assumed that there is no incoming flux $\left\langle J_{v}^{(1)}\right\rangle$ from infinity.

From this example we learn the obvious lesson. We have to assume that the currents $J_{U \ldots U}^{(s)}$ are regular at the horizon in the Kruskal coordinate $U=-e^{-\kappa u}$. Since these currents are covariant, we have

$$
J_{U \ldots U}^{(s)}=\frac{1}{(-\kappa U)^{s}} J_{u \ldots u}^{(s)}(u)
$$

It then follows that the currents $J_{u \ldots u}^{(s)}$, and their $n-1$ derivatives vanish. From (6.8)-(6.10), at the horizon we must get

$$
\begin{aligned}
j_{u u}^{(2)} & =-\lambda\left(2 A_{u}^{2}-\frac{T}{12}\right) \\
j_{\text {uиu }}^{(3)} & =-\lambda\left(\frac{8 A_{u}^{3}}{3}-\frac{A_{u} T}{3}\right) \\
j_{\text {uиuи }}^{(4)} & =-\lambda\left(4 A_{u}^{4}-\frac{7 T A_{u}^{2}}{5}-\frac{2}{5}\left(\nabla_{u}^{2} A_{u}\right) A_{u}+\frac{7 T^{2}}{240}+\frac{3}{5}\left(\nabla_{u} A_{u}\right)^{2}\right)
\end{aligned}
$$

As already remarked, at infinity the background fields $A_{u}$ and $\phi$ vanish. So that

$$
\left\langle J_{u \ldots u}^{(s)}\right\rangle=\left\langle j_{u \ldots u}^{(s)}\right\rangle_{h}
$$

Now, we evaluate the derivatives on right hand side of (6.20) at the horizon. Setting $\lambda=\hbar=1$ we get

$$
\begin{aligned}
\left\langle j_{u u}^{(2)}\right\rangle_{h} & =\frac{\langle T\rangle_{h}}{12}-\frac{\left\langle A_{t}\right\rangle_{h}^{2}}{2} \\
\left\langle j_{\text {uuu }}^{(3)}\right\rangle_{h} & =-\frac{1}{3}\left\langle A_{t}\right\rangle_{h}^{3}-\frac{1}{6}\langle T\rangle_{h}\left\langle A_{t}\right\rangle_{h} \\
\left\langle j_{\text {uuuu }}^{(4)}\right\rangle_{h} & =-\frac{1}{4}\left\langle A_{t}\right\rangle_{h}^{4}+\frac{1}{4}\langle T\rangle_{h}\left\langle A_{t}\right\rangle_{h}^{2}-\frac{7}{240}\langle T\rangle_{h}^{2}
\end{aligned}
$$

Therefore at infinity we get

$$
\begin{aligned}
-\frac{1}{2 \pi}\left\langle J_{t}^{(2) r}\right\rangle & =\frac{\kappa^{2}}{48 \pi}+\frac{\Omega^{2}}{4 \pi} \\
-\frac{1}{2 \pi}\left\langle J_{t t}^{(3) r}\right\rangle & =\frac{\Omega^{3}}{6 \pi}+\frac{\kappa^{2} \Omega}{24 \pi} \\
-\frac{1}{2 \pi}\left\langle J_{t t t}^{(4) r}\right\rangle & =\frac{7 \kappa^{4}}{1920 \pi}+\frac{\Omega^{2} \kappa^{2}}{16 \pi}+\frac{\Omega^{4}}{8 \pi}
\end{aligned}
$$

where we have used $\left\langle f^{\prime}\left(r_{+}\right)\right\rangle_{h}=2 \kappa,\langle T\rangle_{h}=-\frac{\kappa^{2}}{2}, A_{t}(r)=\frac{r}{r^{2}+a^{2}}, \Omega=A_{t}\left(r_{+}\right)=\left\langle A_{t}\right\rangle_{h}$. These results agree with formula (4.4) after the replacement $A_{t} \rightarrow m A_{t}$ (see the comment before eq.(6.17). We have checked, [2], the agreement up to spin 8 current $\left\langle J_{t \ldots .}^{(8) r}\right\rangle$. 


\section{Anomalies and higher spin currents}

Each higher spin current carries to infinity its own component of the Hawking radiation. Just in the same way as in the action the metric is a source for the energy-momentum tensor, these new (covariant) currents will have in the effective action suitable sources, with the appropriate indices and symmetries. We will represent such sources by means of background fields $B_{\mu_{1} \ldots \mu_{s}}^{(s)}$ (which will be eventually set to zero). So we have

$$
J_{\mu_{1} \ldots \mu_{s}}^{(s)}=\frac{1}{\sqrt{g}} \frac{\delta}{\delta B^{(s) \mu_{1} \ldots \mu_{s}}} S
$$

We assume that all $J_{\mu_{1} \ldots \mu_{s}}^{(s)}$ are maximally symmetric and classically traceless ${ }^{2}$.

In the previous section the covariant forms of the higher spin currents have not given rise to any trace or diffeomorphisms or gauge anomaly. One might object that this may be due to the particular currents we are considering and attribute it to an accident. For instance, in [17], the authors, using higher spin currents defined by different combinations of the $j_{z \ldots z}^{(m, n)}$ from (5.1), did find anomalies. So the question is: is there a way to spell out this ambiguity? The answer is yes, this can be done by means of cohomological (or consistency) methods applied to the effective field theory.

Let us concentrate here, for simplicity, on trace anomalies. The much more complicated analysis of diff anomalies has been carried out in [2] and will not be reviewed here. The consistency conditions for trace anomalies are similar to the Wess-Zumino consistency conditions for chiral anomalies and are based on the simple remark that, if we apply two symmetry transformations in different order to the action, the result must obey the group theoretical rules of the transformations. In particular, since Weyl transformations are Abelian, making two Weyl transformations in opposite order must bring the same result. Although this explains the geometrical meaning of the consistency conditions, proceeding in this way is often very cumbersome. The method becomes more manageable if we transform it into a cohomological problem. This is simple: just promote the local transformation parameters to anticommuting fields (ghosts). The transformations become nilpotent and define a coboundary operator. It is in general possible to compute the non-trivial cocycles of such coboundary operators: they are local polynomials of the fields and their derivatives, and they define the possible anomalies. This cohomological method however does not guarantee that a given cocycle makes its appearance on the right hand side of a specific conservation law, and consequently violates it, for the numerical coefficient in front of it may vanish. Therefore this method by itself is not enough to fully determine anomalous conservation laws. On the other hand it is very powerful if we can show that all the cocycles (that is, all the potential anomalies) are trivial. In this case we are guaranteed that the corresponding conservation law will not be broken by anomalies, or, if they are, the corresponding (trivial) anomalies can be absorbed by a redefinition of the action.

We notice that the cohomological analysis of anomalies does not depend on whether we are dealing with fermionic or bosonic currents, but only on the background fields. This said we can now analyze the problem of the existence of trace anomalies in higher spin currents with cohomological

\footnotetext{
${ }^{2}$ Other background fields, beside the $B$ ones, are needed in the effective action, as was discussed in [2]. But they turn out not to be irrelevant in the anomaly discussion, so we will drop them here.
} 
methods. The relevant background is formed by the metric, the completely symmetric $B$ fields and the electromagnetic field.

Of course the electromagnetic field gives rise to the gauge anomaly in the covariant derivative of the $J^{(1)}$ current, see (6.11). The latter is induced by the gauge transformation $\delta_{\lambda} A_{\mu}=\partial_{\mu} \lambda$ and this exhausts our discussion of the gauge anomaly.

Next let us turn to the trace anomalies and recall the appropriate setting for this type of analysis. We start from the analysis of $J^{(3)}$. Setting $B_{\mu \nu \lambda}^{(3)}=B_{\mu \nu \lambda}$ the Weyl transformation of the various field involved are

$$
\begin{aligned}
\delta_{\sigma} g_{\mu \nu} & =2 \sigma g_{\mu \nu} \\
\delta_{\sigma} B_{\mu \nu \lambda} & =x \sigma B_{\mu \nu \lambda} \\
\delta_{\sigma} A_{\mu} & =0
\end{aligned}
$$

which induces the trace of the energy-momentum tensor, and

$$
\begin{aligned}
\delta_{\tau} g_{\mu \nu} & =0 \\
\delta_{\tau} B_{\mu \nu \lambda} & =\tau_{\mu} g_{\nu \lambda}+\mathrm{cycl} \\
\delta_{\tau} A_{\mu} & =0
\end{aligned}
$$

which induces the trace of $J^{(3)}$. Moreover, for consistency with (7.2), we must have

$$
\delta_{\sigma} \tau_{\mu}=(x-2) \sigma \tau_{\mu}
$$

where $x$ is an arbitrary number.

A comment on these transformations is in order. They are determined as follows: they must be expressed in terms of symmetry parameters and of the basic background fields $g_{\mu \nu}$ and $A_{\mu}$ and nothing else; they must form a Lie algebra, as was mentioned above, and they must leave unchanged the terms in the effective action, in particular the terms involving the matter fields. The transformations are then dictated by the canonical dimensions of the various fields. The fields $B^{(s)}$ have dimension $2-s$.

We must now repeat the analysis we have done in [1]. We promote $\sigma$ and $\tau_{\mu}$ to anticommuting fields so that

$$
\delta_{\sigma}^{2}=0, \quad \delta_{\tau}^{2}=0, \quad \delta_{\sigma} \delta_{\tau}+\delta_{\tau} \delta_{\sigma}=0
$$

Integrated anomalies are defined by

$$
\delta_{\sigma} \Gamma^{(1)}=\hbar \Delta_{\sigma}, \quad \delta_{\tau} \Gamma^{(1)}=\hbar \Delta_{\tau},
$$

where $\Gamma^{(1)}$ is the one-loop quantum action and $\Delta_{\sigma}, \Delta_{\tau}$ are local functional linear in $\sigma$ and $\tau$, respectively. The unintegrated anomalies, i.e. for instance the traces $T_{\mu}^{\mu}$ and $J^{(3) \mu}{ }_{\mu \lambda}$ are obtained by functionally differentiating with respect to $\sigma$ and $\tau_{\lambda}$, respectively.

By applying $\delta_{\sigma}, \delta_{\tau}$ to the eqs.(7.7), we see that candidates for anomalies $\Delta_{\sigma}$ and $\Delta_{\tau}$ must satisfy the consistency conditions

$$
\delta_{\sigma} \Delta_{\sigma}=0, \quad \delta_{\tau} \Delta_{\sigma}+\delta_{\sigma} \Delta_{\tau}=0, \quad \delta_{\tau} \Delta_{\tau}=0
$$


Once we have determined these cocycles we have to make sure that they are true anomalies, that is that they are nontrivial. In other words there must not exist local counterterm $C$ in the action such that

$$
\begin{aligned}
& \Delta_{\sigma}=\delta_{\sigma} C \equiv \delta_{\sigma} \int d^{2} x \sqrt{-g} \mathcal{C} \\
& \Delta_{\tau}=\delta_{\tau} C \equiv \delta_{\tau} \int d^{2} x \sqrt{-g} \mathcal{C}
\end{aligned}
$$

If such a $C$ existed we could redefine the quantum action by subtracting these counterterms and get rid of the (trivial) anomalies.

Let us consider now the problem of the trace $J^{(3) \mu}{ }_{\mu \lambda}$. We could repeat the complete analysis of [1], but there is a shortcut due to the simple form of the transformations (7.4). Suppose we find cocycle $\Delta_{\tau}^{(3)}$

$$
\Delta_{\tau}^{(3)}=\int d^{2} x \sqrt{-g} \tau^{\mu} I_{\mu}^{(3)}
$$

where $I_{\mu}^{(3)}$ is a canonical dimension 3 tensor made of the metric, the gauge field and their derivatives, such as $\nabla_{\mu} R$ or $\nabla_{\nu} F_{\mu}{ }^{\nu}$, or even a non-gauge-invariant tensor such as $A_{\mu} R$. Then it is immediate to write down a counterterm

$$
\mathcal{C}^{(3)} \sim B_{\lambda}^{\mu \lambda} I_{\mu}^{(3)}
$$

which cancels (7.11).

As for the trace $J^{(4) \mu}{ }_{\nu \lambda \rho}$ we can proceed in analogy to $J^{(3) \mu}{ }_{\mu \lambda}$. Setting $B_{\mu \nu \lambda \rho}^{(4)} \equiv B_{\mu \nu \lambda \rho}$, the relevant Weyl transformations are as follows. The variation $\delta_{\tau}$ acts only on $B_{\mu \nu \lambda \rho}$

$$
\delta_{\tau} B_{\mu \nu \lambda \rho}=g_{\mu \nu} \tau_{\lambda \rho}+\mathrm{cycl}
$$

and the other fields remain unchanged while the variation with respect to the ordinary Weyl parameter $\sigma$ are

$$
\begin{aligned}
& \delta_{\sigma} g_{\mu \nu}=2 \sigma g_{\mu \nu} \\
& \delta_{\sigma} \tau_{\mu \nu}=(x-2) \sigma \tau_{\mu \nu} \\
& \delta_{\sigma} B_{\mu \nu \lambda \rho}=x \sigma B_{\mu \nu \lambda \rho}
\end{aligned}
$$

where, again, $x$ is an arbitrary number. Now we can repeat the previous argument. Let a cocycle have the form

$$
\Delta_{\tau}^{(4)}=\int d^{2} x \sqrt{-g} \tau^{\mu \nu} I_{\mu \nu}^{(4)}
$$

where $I_{\mu}^{(4)}$ is a dimension 4 tensor made out of the metric, the gauge field and their derivatives, such as $\nabla_{\mu} \nabla_{\nu} R$. The counterterm

$$
\mathcal{C}^{(4)} \sim B_{\lambda}^{\mu \nu \lambda} I_{\mu \nu}^{(4)}
$$

cancels (7.17). 
It is not hard to generalize this conclusion to higher spin currents.

Out of conciseness the analysis in this section has been somewhat oversimplified. A more complete analysis of trace anomalies can be found in [1] and, as far as the diffeomorphisms anomalies are concerned, in [2]. See also complementary considerations in [3]. On the basis of these analysis we conclude on a general ground that anomalies may not arise in the spin higher than 2 currents under any condition, or if they arise they are trivial.

\section{Conclusion}

It is evident that the possibility to describe the higher moments of the Hawking radiation is related to the transformation properties of the holomorphic higher spin currents, specifically to the appearance of generalized Schwarzian derivatives in their conformal transformation properties. Even in the case of the energy momentum tensor, the Hawking flux is related to Weyl or Diff anomalies only in the sense that the latter determine the relation between the covariant and holomorphic part of the energy-momentum tensor (see our discussion in [2]). For higher spin currents, as we have seen, there are no links with anomalies simply because anomalies cannot exist in the conservation laws of these currents. This much seems definitely clear. There are however other aspects of the problem which have remained so far implicit, but are crucial in order to understand the central role of the $W_{1+\infty}$ algebra. In this section we would like to discuss these aspects.

Let us start from the remark that the summation over $k$ in formula (5.1) does not affect the crucial term $\frac{\kappa^{s}}{s}\left(1-2^{-(s-1)}\right) B_{s}$ in $\left\langle X_{s}^{F}\right\rangle$ except for an overall multiplicative factor. This means that, had we used each one of the currents

$$
j_{z \ldots z}^{(s, k)}(z)=: \partial_{z}^{s-k} \Psi^{\dagger}(z) \partial_{z}^{k-1} \Psi(z):
$$

instead of (5.1), we would have obtained (up to normalization) the same final result for the moments of the Hawking radiation. This seems at first to deprive of any interest the role of the $W_{1+\infty}$ algebra, but the case is just the opposite. Using the currents $j_{z \ldots z}^{(s, k)}(z)$ we have two enormous disadvantages.

The first is that we do not have any means of normalizing these currents, thus rendering the results obtained by their means devoid of any predictive value. The $W_{1+\infty}$ algebra structure tells us how to normalize the currents in such a way as to get an algebra. There remain only two constants to be fixed $\lambda$ and $q$. The first is fixed in such a way as to get the right transformation laws (OPE) of the energy-momentum tensor, the second is fixed by the $\mathrm{U}(1)$ algebra of $j^{(1)}$. Once these two constants are fixed the normalization for all the higher spin currents is uniquely determined and in agreement with the Planckian spectrum of the Hawking radiation.

The second disadvantage of using currents that do not form a $W_{1+\infty}$ algebra, such as $j_{z \ldots z}^{(s, k)}(z)$, is the appearance of anomalies in their traces or in the conservation laws of their covariant version. This was shown in a very explicit way in [17]. As we have shown, these anomalies are cohomologically trivial and can be eliminated by suitable redefinitions or subtractions. As a result one ends up with the currents (5.1) and their $W_{1+\infty}$ algebra. In other words the $W_{1+\infty}$ algebra is the appropriate structure underlying the Planckian spectrum of the Hawking radiation.

It goes without saying that all these conclusions can be drawn also for the higher spin bosonic currents and the corresponding $W_{\infty}$ algebra. 
This result seems to entail a dramatic consequence. It has been known since long that the dynamics near the horizon of a black hole is conformal. What we learn from the above analysis is that the two-dimensional physics around the horizon is characterized by a symmetry larger than the Virasoro algebra, such as a $W_{\infty}$ or $W_{1+\infty}$ algebra.

Finally there is an important facet of our results which is implicit in what we have said above, but deserves to be spelled out more explicitly. The existence of (true) anomalies in higher spin currents or, alternatively, the lack of an algebraic principle in the normalization of such currents, would have meant the existence of 'hairs' corresponding the higher spin charges, thus violating the 'no hair' theorem that must characterize the Hawking radiation. Therefore our conclusions are in nice agreement with this theorem.

\section{References}

[1] L. Bonora and M. Cvitan, Hawking radiation, $W_{\infty}$ algebra and trace anomalies, JHEP 0805 (2008) 071 [arXiv:0804.0198 [hep-th]].

[2] L. Bonora, M. Cvitan, S. Pallua and I. Smolić, Hawking Fluxes, $W_{\infty}$ algebra and anomalies, JHEP 0812 (2008) 021 [arXiv:0808.2360 [hep-th]].

[3] L. Bonora, M. Cvitan, S. Pallua and I. Smolic, Hawking fluxes, Fermionic currents, W(1+infinity) algebra and anomalies, arXiv:0907.3722 [hep-th].

[4] S. W. Hawking, Particle Creation By Black Holes, Commun. Math. Phys. 43, 199 (1975) [Erratum-ibid. 46, 206 (1976)].

[5] G. W. Gibbons and S. W. Hawking, Action Integrals And Partition Functions In Quantum Gravity, Phys. Rev. D 15, 2752 (1977).

[6] S. M. Christensen and S. A. Fulling, Trace Anomalies And The Hawking Effect, Phys. Rev. D15 (1977) 2088.

[7] P.C.W.Davies, S.A.Fulling and W.G.Unruh, Energy Momentum Tensor Near An Evaporating Black Hole, Phys. Rev. D13 (1976) 2720.

[8] L. Thorlacius, Black hole evolution, Nucl. Phys. Proc. Suppl. 41 (1995) 245 [arXiv:hep-th/9411020].

[9] A. Strominger, Les Houches lectures on black holes, arXiv:hep-th/9501071.

[10] S. P. Robinson and F. Wilczek, A relationship between Hawking radiation and gravitational anomalies, Phys. Rev. Lett. 95 (2005) 011303 [arXiv:gr-qc/0502074].

[11] R. Banerjee and S. Kulkarni, Hawking Radiation, Effective Actions and Covariant Boundary Conditions, Phys. Lett. B 659 (2008) 827 [arXiv:0709.3916 [hep-th]].

[12] E. Bergshoeff, C.N. Pope, L.J. Romans, E. Sezgin, X. Shen: The super $W_{\infty}$ algebra, Phys. Lett. B 245 (1990) 447.

[13] S. Iso, H. Umetsu and F. Wilczek, Anomalies, Hawking radiations and regularity in rotating black holes, Phys. Rev. D 74 (2006) 044017 [arXiv:hep-th/0606018].

[14] S. Iso, T. Morita and H. Umetsu, Quantum anomalies at horizon and Hawking radiations in Myers-Perry black holes, JHEP 0704 (2007) 068 [arXiv:hep-th/0612286].

[15] S. Iso, T. Morita and H. Umetsu, Higher-spin currents and thermal flux from Hawking radiation, Phys. Rev. D 75 (2007) 124004 [arXiv:hep-th/0701272]. 
[16] S. Iso, T. Morita and H. Umetsu, Fluxes of Higher-spin Currents and Hawking Radiations from Charged Black Holes, Phys. Rev. D 76 (2007) 064015 [arXiv:0705.3494 [hep-th]].

[17] S. Iso, T. Morita and H. Umetsu, Higher-spin Gauge and Trace Anomalies in Two-dimensional Backgrounds, Nucl. Phys. B 799 (2008) 60 [arXiv:0710.0453 [hep-th]].

[18] S. Iso, T. Morita and H. Umetsu, Hawking Radiation via Higher-spin Gauge Anomalies, Phys. Rev. D 77 (2008) 045007 [arXiv:0710.0456 [hep-th]]. 